\title{
Scattering from Impurities in a Crystal
}

\author{
F. Bentosela* \\ Université d'Aix-Marseille II, U.E.R. de Luminy \\ et Centre de Physique Théorique, CNRS Marseille, Marseille, France
}

\begin{abstract}
A time independent scattering theory for a particle in a crystal with impurity is given. It is shown that the scattered wave is the solution of a Lippman Schwinger equation, and that the existence of bound states or narrow resonances is related more to the band structure than to the form of the impurity potential.
\end{abstract}

\section{Introduction}

This paper is concerned with the scattering of wavelike excitations in solids by localized imperfections: $Q(x)^{1}$. We extend the treatment of the two body quantum mechanics by Kato and Kuroda [1,2] and prove the existence of distorded Bloch waves which have the form (Bloch wave) + (outgoing wave)/(incoming wave) and are obtained as solutions of a Lippman-Schwinger integral equation.

We want to emphasize, here, the role played by the critical energies ${ }^{2}$. In ordinary scattering the only critical energy is 0 and this point can be an accumulation point for the eigenvalues for instance if the impurity potential is $Q(x)=|x|^{-1}$. In our case critical energies can be embedded in the bands which form the continuous spectrum of $H_{B}=-\Delta+V$, and we prove that these points can be also accumulation points for the eigenvalues but now this phenomena originates more from the band structure than from the nature of the potential. As eigenvalues or

* Postal address: Centre de Physique Théorique, C.N.R.S., 31, chemin Joseph Aiguier, F-13274 Marseille Cedex 2, France.

1 Scattering of electrons by foreign atoms, phonons by mass defects or spin waves by magnetic defects can be studied simultaneously with slight modifications (see Callaway [18]).

2 Let $V$ be the periodic potential: $V(\boldsymbol{x}+\boldsymbol{R})=V(\boldsymbol{x})$ if $\boldsymbol{R} \in \mathbb{L} \sim \mathbb{Z}^{3}$ and suppose $\int_{\mathbb{R}^{3} / \mathbb{L}}|V(\boldsymbol{x})|^{2} d^{3} x<+\infty$. Call $\mathbb{L}^{\perp}=\left\{\boldsymbol{K} \in \mathbb{R}^{3} \mid \boldsymbol{K} \cdot \boldsymbol{R}=2 \pi n, \forall \boldsymbol{R} \in \mathbb{L}\right\}$, the reciprocical lattice and $\mathbb{B}=\mathbb{R}^{3} / \mathbb{L}^{\perp}$ the Brillouin zone; $H_{B}=\Delta+V$ can be decomposed in a direct integral $\int_{B}^{\oplus} H_{B}(k) d^{3} k ; H_{B}(k)$ acts on $\ell^{2}$; it is proven in [14] that the spectrum of $H_{B}(k)$ is discrete and the set of eigenvalues is noted $\left\{E_{1}(k), E_{2}(k), \ldots, E_{n}(k), \ldots\right\}$ and the $n^{\text {th }}$ eigenvector: $\left\{c_{n}^{\mathbf{K}}(k)\right\} \in \ell^{2}$. The critical points of $E_{n}(k)$ are the $k \in \mathbb{B}$ such that $\nabla E_{n}(k)=0$, the values of $E_{n}$ at these points are the critical energies. 
more generally narrow resonances near the critical energies enlarge the differential cross section and accordingly the electrical resistivity, measurements can give experimental evidence for their existence (Kosicki and Paul, 1966, [3]). Optical transitions have also been observed (Onton, 1971, [4]) from the impurity ground state to resonant states.

Other attempts try to give a theoretical basis to this phenomena in particular the papers by Bassani, Iadonisi and Preziosi [5-7] whose approach is based on the effective mass approximation applied to the secondary minima.

In this paper we get complete results for the scattering theory of the operator: $H=-\Delta+V+Q=H_{B}+Q$.

Existence of time dependent wave operators has been studied previously by Lenahan (Thesis, 1970, [11]) where $V$ is a bounded periodic function, and the impurity $Q \in L^{1}\left(\mathbb{R}^{3}\right) \cap L^{2}\left(\mathbb{R}^{3}\right)$; by Thomas $(1973$, [10]) where $V$ is square integrable over a unit cell, $\square \equiv \mathbb{R}^{3} / \mathbb{L}: \int_{\square}|V(x)|^{2} d^{3} x<+\infty$ and $Q$ is as before. Kuroda [2] and Troianiello $(1974,[12])$ gave an eigenfunction expansion when $V$ is a bounded continuous function and $Q \in L^{1}\left(\mathbb{R}^{3}\right) \cap L^{2}\left(\mathbb{R}^{3}\right)$. This paper improves the former ones since it gives simultaneously the two results with: $\int_{\square}|V(x)|^{2} d^{3} x<+\infty$; $Q \in L^{2,{ }^{l o c}}\left(\mathbb{R}^{3}\right)$ and $Q(x) \rightarrow 1 /|x|^{2+\varepsilon}$ as $|x| \rightarrow+\infty$.

In the first section we utilize the estimates given, in Appendix A, on the resolvent kernel or Green's function of $H_{B}$, for studying the operators $Q_{2}\left(H_{B}-\lambda\right)^{-1} Q_{1}$ (where $Q=Q_{1} Q_{2}$ ) and its limit when $\lambda$ goes to an energy belonging to the continuous spectrum of $H_{B}$, and we prove the existence and completeness of the wave operators under the hypothesis on $V$ and $Q$ above mentioned. In Section 2, with the same $V$ and $Q$ we construct a generalized eigenfunction expansion along the lines of Ikebe [8], Simon [9], Kato and Kuroda [1,2]. In Section 3, we prove that only critical energies can be accumulation points for the eigenvalues if $Q$ is short range.

\section{Conditions on Periodic and Impurity Potentials for Having the Existence and Completness of the Wave Operators}

The first fundamental theorem by Kato and Kuroda can be adapted as follows (see Guillot [13]).

Denote $Q_{1}(\boldsymbol{x})=1 /\left(1+|\boldsymbol{x}|^{3 / 2+\varepsilon}\right)$ and write $Q(\boldsymbol{x})=Q_{1}(\boldsymbol{x}) Q_{2}(\boldsymbol{x})$. Put:

$$
\mathscr{H}^{+}=\left\{f \mid\left(1+|\boldsymbol{x}|^{3 / 2+\varepsilon}\right) f \in L^{2}\left(\mathbb{R}^{3}\right)\right\} \text {. }
$$

Theorem Kato-Kurado 1.1. Let $\Gamma$ a Borel set of the real axis and suppose that the following conditions hold:

Condition I.1. For each $E \in \Gamma$

$$
\lim _{\varepsilon \rightarrow 0}\left(\left\{\left(H_{B}-E-i \varepsilon\right)^{-1}-\left(H_{B}-E+i \varepsilon\right)^{-1}\right\} f, g\right)
$$

exists for each $(f, g) \in \mathscr{H}^{+} \times \mathscr{H}^{+}$or equivalently:

$$
\lim _{\varepsilon \rightarrow 0}\left(f,\left[Q_{1}\left(H_{B}-E-i \varepsilon\right)^{-1} Q_{1}-Q_{1}\left(H_{B}-E+i \varepsilon\right)^{-1} Q_{1}\right] g\right)
$$

exists for each $(f, g) \in L^{2}\left(\mathbb{R}^{3}\right) \times L^{2}\left(\mathbb{R}^{3}\right)$. 
Condition I.2. For every $\varepsilon>0$

$s-\lim _{\varepsilon \rightarrow 0} Q\left(H_{B}-(E \pm i \varepsilon)\right)^{-1}$

belong to $\mathscr{C}\left(\mathscr{H}^{+}\right)$: set of compact operators in $\mathscr{H}^{+}$or equivalently:

$$
A\left(E_{ \pm}\right) \equiv s-\lim _{\varepsilon \rightarrow 0} Q_{2}\left(H_{B}-(E \pm i \varepsilon)\right)^{-1} Q_{1}
$$

belong to $\mathscr{C}\left(L^{2}\left(\mathbb{R}^{3}\right)\right)$.

Condition I.3.

$$
\left(1+A\left(E_{ \pm}\right)\right)^{-1} \text { exists } \forall E \in \Gamma \text {. }
$$

Then:

1. The absolute continuous part of $H$ is unitarily equivalent to the a.c. part of $H_{B}$ on $\Gamma$; i.e. it exists $U_{ \pm}$unitary such that

$$
E_{\mathrm{ac}}(\Gamma) H=U_{ \pm} E_{B, \mathrm{ac}}(\Gamma) H_{B} U_{ \pm}
$$

$E_{\mathrm{ac}}(\Gamma), E_{B, \mathrm{ac}}(\Gamma)$ are the absolute continuous part of the spectral measure associated res. with $H=H_{B}+Q$ and $H_{B}$.

2. The time dependent wave operators

$$
W_{I}=s-\lim _{t \rightarrow \pm \infty} e^{i t H} e^{-i t H_{B}} E_{B, \text { ac }}(\Gamma)
$$

exist and are complete.

Now we have to put conditions on $V$ and $Q$ such that Conditions I.1-3 be verified.

Theorem 1.2. If $V$ satisfies $\int_{\square}|V(\boldsymbol{x})|^{2} d^{3} x<+\infty \Leftrightarrow \sum_{\boldsymbol{K}}|\hat{V}(\boldsymbol{K})|^{2}<+\infty$ and $Q_{2}(x)=$ $\left(1+|x|^{3 / 2+\varepsilon}\right) Q(x)$ is the sum of an $L^{2}$ function and an $L^{6}$ function (for instance $Q$ can be $\sim 1 /|x|^{3-\eta}$ at the origin and $\sim 1 /|x|^{2+\varepsilon}$ at infinity).

Then Conditions $1.1-3$ are verified for whatever $\Gamma$ contained in $\mathbb{R}^{1}-\left(\mathscr{E}_{c}^{-} \cup \mathscr{S}\right)$ where $\mathscr{E}_{c}^{-} \subset \mathscr{E}_{c}$ (critical energies), and $\mathscr{S}$ is a closed set with zero measure.

First recall some spectral properties of $H_{B}$ (see Avron, Grossmann and Rodriguez [14]); functions $k \in \mathbb{B} \rightarrow E_{n}(k) \in \mathbb{R}^{1}$ are differentiable and holomorphic in the three variables $k_{1}, k_{2}, k_{3}$ in the regions of $\mathbb{B}$ where the bands do not intersect.

The improper eigenfunctions of $H_{B}$, called Bloch functions ${ }^{3}, \psi_{n \boldsymbol{k}}^{B}(x)=$ $\sum_{\boldsymbol{K}} c_{n}^{\boldsymbol{K}}(k) \exp (i(k+\boldsymbol{K}) x)$ have the following properties: they have the same holomorphic properties in $k$ as the $E_{n}(k)$; it can be proven (see Appendix B) that $\left\{c_{n}^{\mathbf{K}}(k)\right\} \in \ell^{1} \cap \ell^{2}$, then $\sum_{\boldsymbol{K}} c_{n}^{\mathbf{K}_{n}}(\boldsymbol{k}) \exp (i \boldsymbol{K} x)$ is a bounded periodic function.

Consider a Borel set $\Lambda$ and denote by $\boldsymbol{I}$ the set of bands, $n$, such that $E_{n}(k) \in \Lambda$ for some $k \in \mathbb{B}$. If the diameter of $\Lambda$ is finite, $\boldsymbol{I}$ is also a finite set (cf. Thomas [10],

$3 \quad$ Bloch functions are also the kernel of the unitary operator $U_{B}$ from $L^{2}\left(\mathbb{R}^{3}\right)$ to $L^{2}(\mathbb{N} \times \mathbb{B})$ which diagonalizes the Bloch hamiltonian: $U_{B} H_{B} U_{B}^{-1}$ is the multiplication by $E_{n}(k)$;

$$
\left(U_{B} f\right)(n, k)=\int_{\mathbb{R}^{3}} \psi_{n k}^{B^{*}}(\boldsymbol{x}) f(\boldsymbol{x}) d^{3} x .
$$




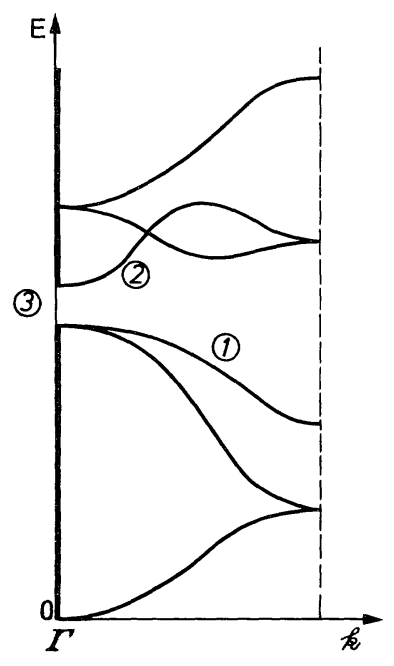

Fig. 1. Energy band of $G e$ estimated by Hermann in the $(1,0,0)$ direction. 1 valence band, 2 conduction band, 3 gaps between the bands

Lemma A.2). Call $P_{\boldsymbol{I}}=\sum_{n \in \boldsymbol{I}} \int^{\oplus} P_{n}(k) d^{3} k$, where $P_{n}(k)$ is the one-dimensional projector in $\ell^{2}$ corresponding to the $n^{\text {th }}$ eigenvector of $H_{B}(k):\left\{c_{n}^{\mathbf{K}}(k)\right\}$.

The proof of Theorem 1.2 will be separated in two parts: the first one is concerned by $Q_{2} P_{I}\left(H_{B}-E_{ \pm}\right)^{-1} Q_{1}$ (Lemma 1.3$)$, the second one by $Q_{2}\left(1-P_{I}\right)\left(H_{B}-\right.$ $E)^{-1} Q_{1}$ (Lemma 1.4).

Lemma 1.3. If $Q_{2}(x)=\left(1+|x|^{3 / 2+\varepsilon}\right) Q(x) \in L^{p}$ with $2 \leqq p \leqq 6$ and $E \in \Lambda-\mathscr{E}_{c}^{-}$.

a) $Q_{2} P_{\boldsymbol{I}}\left(H_{B}-E_{ \pm}\right)^{-1} Q_{1}$ is an Hilbert-Schmidt operator.

b) It is continuous in E relative to the Hilbert-Schmidt norm.

Proof. a) Let $G_{B}^{\boldsymbol{I}}(\boldsymbol{x}, \boldsymbol{y} ; E \pm i \varepsilon)$ designates the kernel of $P_{\boldsymbol{I}}\left(H_{0}-E \mp i \varepsilon\right)^{-1}$

$$
G_{B}^{\boldsymbol{I}}(\boldsymbol{x}, \boldsymbol{y} ; E \pm i \varepsilon)=\sum_{n \in \boldsymbol{I}} \int_{\mathbb{B}} d^{3} k \frac{\psi_{n k}(\boldsymbol{x}) \psi_{n k}^{*}(\boldsymbol{y})}{E_{n}(k)-E \mp i \varepsilon} .
$$

Write $\boldsymbol{x}=\boldsymbol{x}_{0}+\boldsymbol{R}_{1}$ and $\boldsymbol{y}=\boldsymbol{y}_{0}+\boldsymbol{R}_{2}$ with $\boldsymbol{x}_{0}$ and $\boldsymbol{y}_{0}$ belonging to the first direct cell, $\square$. then:

$$
G_{B}^{\boldsymbol{I}}(\boldsymbol{x}, \boldsymbol{y} ; E \pm i \varepsilon)=\sum_{n \in \boldsymbol{I}} \int d^{3} k \exp \left(i k\left(\boldsymbol{R}_{1}-\boldsymbol{R}_{2}\right)\right) \frac{\psi_{n \boldsymbol{k}}\left(\boldsymbol{x}_{0}\right) \psi_{n \boldsymbol{k}}^{*}\left(\boldsymbol{y}_{0}\right)}{E_{n}(k)-E \mp i \varepsilon} \equiv g_{\boldsymbol{R}_{1}-\boldsymbol{R}_{2}}\left(\boldsymbol{x}_{0}, \boldsymbol{y}_{0}, E \pm i \varepsilon\right) .
$$

In Appendix A we prove that $\left|\lim _{\varepsilon \rightarrow 0} g^{\boldsymbol{R}}\left(\boldsymbol{x}_{0}, \boldsymbol{y}_{0}, E \pm i \varepsilon\right)\right| \leqq \alpha \pm(E) /(1+|\boldsymbol{R}|)$ if $E \notin \mathscr{E}_{c}^{-}$.

Consider now the Hilbert-Schmidt norm of $Q_{2} P_{\boldsymbol{I}}\left(H_{B}-E_{ \pm}\right)^{-1} Q_{1}$ :

$$
\begin{aligned}
& \equiv \iint d^{3} x d^{3} y\left|Q_{1}(\boldsymbol{y})\right|^{2}\left|Q_{2}(\boldsymbol{x})\right|^{2}\left|G_{B}^{\boldsymbol{I}}\left(\boldsymbol{x}, \boldsymbol{y}, E_{ \pm}\right)\right|^{2} \\
& =\int_{\square} \int_{\square} d^{3} x_{0} d^{3} y_{0} \sum_{\boldsymbol{R}_{1}}\left|Q_{2}\left(\boldsymbol{x}_{0}+\boldsymbol{R}_{1}\right)\right|^{2} \sum_{\boldsymbol{R}_{2}}\left|Q_{1}\left(\boldsymbol{y}_{0}+\boldsymbol{R}_{2}\right)\right|^{2}\left|g_{\boldsymbol{R}_{1}-\boldsymbol{R}_{2}}\left(\boldsymbol{x}_{0}, \boldsymbol{y}_{0}, E_{ \pm}\right)\right|^{2} .
\end{aligned}
$$

The convolution of the two series $\left\{\left|Q_{1}\left(\boldsymbol{y}_{0}+\boldsymbol{R}\right)\right|^{2}\right\} \in \ell^{q / 2}$ with $2 \leqq q \leqq \infty$ and $\left\{\left|g_{\boldsymbol{R}}\left(\boldsymbol{x}_{0}, \boldsymbol{y}_{0}, E_{ \pm}\right)\right|^{2}\right\} \in \ell^{3 / 2+\varepsilon}$ belongs to $\ell^{p^{\prime}}$ with $1 / p^{\prime}=2 / q+1 /(3 / 2+\varepsilon)-1$ if $q \leqq 6$. If 
$Q_{2} \in L^{p},\left\{\left|Q_{2}\left(\boldsymbol{x}_{0}+\boldsymbol{R}\right)\right|^{2}\right\} \in \ell^{p / 2}$ for a.e., $\boldsymbol{x}_{0}$ then the total is finite if:

$$
1 / p^{\prime}+2 / p=1 \Leftrightarrow p=3 q /(4 q-3)
$$

i.e. if $p \in[2,6]$. If $p$ belongs to this interval the integrand can be majorized by

$$
\sum_{\boldsymbol{R}_{1}}\left|Q_{1}\left(\boldsymbol{y}_{0}+\boldsymbol{R}_{1}\right)\right|^{2 q} \cdot \sum_{\boldsymbol{R}_{2}}\left|Q_{2}\left(\boldsymbol{x}_{0}+\boldsymbol{R}_{2}\right)\right|^{p} \cdot \sum_{\boldsymbol{R}} \alpha_{ \pm}(E) /(1+|\boldsymbol{R}|)^{3+\varepsilon}
$$

then $(1) \leqq \sum_{\boldsymbol{R}} \alpha_{ \pm}(E) /(1+|\boldsymbol{R}|)^{3+\varepsilon} \int d^{3} y\left|Q_{1}(\boldsymbol{y})\right|^{2 q} \int d^{3} x\left|Q_{2}(\boldsymbol{x})\right|^{p}$. Since by hypothesis $Q_{2} \in L^{p} ; p \in[2,6],(1)$ is finite.

b) The continuity in the Hilbert-Schmidt norm of $Q_{2} P_{I}\left(H_{B}-E_{ \pm}\right)^{-1} Q_{1}$ follows easily from the continuity of $g_{\boldsymbol{R}}\left(\boldsymbol{x}_{0}, \boldsymbol{y}_{0}, E_{ \pm}\right)$(see Appendix A) for all the $E \notin \mathscr{E}_{c}^{-}$.

Lemma 1.4. If $\int|V(\boldsymbol{x})|^{2} d^{3} x<+\infty$ and if $Q_{2}$ is relatively compact ${ }^{4}$ with respect to $-\Delta$, then $Q_{2}\left(1-P_{\mathbf{I}}\right)\left(H_{B}-E\right)^{-1} Q_{1}$ is compact and continuous in $E \in \Lambda$.

Proof. By the spectral theorem: $H_{B}=\sum_{n=1}^{\infty} \int d^{3} k E_{n}(k) P_{n}(k)$ consequently:

$$
\left(1-P_{I}\right)\left(H_{B}-E\right)=H_{B}-E-\sum_{n \in I} \int_{\mathbb{B}} d^{3} k\left(E_{n}(k)-E\right) P_{n}(k) .
$$

\section{Denote}

$$
V_{P S}=V-(E+1)-\sum_{n \in I} \int_{\mathbb{B}} d^{3} k\left(E_{n}(k)-E\right) P_{n}(k) ;
$$

then

$$
\left(1-P_{I}\right)\left(H_{B}-E\right)=-\Delta+V_{P S}+1 \Rightarrow\left(1-P_{I}\right)\left(H_{B}-E\right)^{-1}=\left(-\Delta+V_{P S}+1\right)^{-1} .
$$

Utilize the resolvent equation:

$$
\left(-\Delta+V_{P S}+1\right)^{-1}=(-\Delta+1)^{-1}-\left(-\Delta+V_{P S}+1\right)^{-1} V_{P S}(-\Delta+1)^{-1} .
$$

So

$$
\begin{aligned}
Q_{2}\left(-\Delta+V_{P S}+1\right)^{-1} Q_{1}= & Q_{2}(-\Delta+1)^{-1} Q_{1} \\
& -Q_{2}\left(-\Delta+V_{P S}+1\right)^{-1} V_{P S}(-\Delta+1)^{-1} Q_{1} .
\end{aligned}
$$

a) $Q_{2}(-\Delta+1)^{-1} Q_{1}$ is compact as the product of a compact operator (hypothesis) by a bounded one.

b) From the resolvent equation: $\left(-\Delta+V_{P S}+1\right)^{-1}=(-\Delta+1)^{-1}\left(1+V_{P S}(-\Delta+\right.$ $\left.1)^{-1}\right)^{-1}$. -1 cannot belong to the spectrum of $V_{P S}(-\Delta+1)^{-1}$, in fact, -1 does not belong to the spectrum of $-\Delta+V_{P S}$, as

$E \notin \sigma\left(\left(1-P_{I}\right) H_{B}\right), \quad$ whence $\left(1+V_{P S}(-\Delta+1)^{-1}\right)^{-1}$

is bounded, so, $Q_{2}\left(-\Delta+V_{P S}+1\right)^{-1}$ is compact.

$4 \quad$ A sufficient condition to have $Q_{2}$ relatively compact is $Q_{2}(x) \rightarrow 0$ for $|x| \rightarrow \infty$ and can be expressed as the sum of a $L^{2}$ function and a $L^{\infty}$ one (cf. Kato [15], p. 304, Balslev [16]) improve this result: if $\int_{-y \mid \leq 1}\left|Q_{2}(y)\right|^{2} d^{3} y \rightarrow 0$ for $|x| \rightarrow \infty$ and if $\exists \alpha>0$ such that: $\sup _{x \in \mathbb{R}^{3}} \int\left|Q_{2}(y)\right|^{2}|x-y|^{1-\alpha} d^{3} y<+\infty \Rightarrow Q_{2}(-\Delta+1)^{-1}$ is compact. Potentials $Q_{2}(\boldsymbol{x})=|\boldsymbol{x}|^{-\beta}$ with $0<\beta<\frac{3}{2}$ are relatively compact. 
c) Now it suffices to prove that $V_{P S}(-\Delta+1)^{-1} Q_{1}$ is bounded or more simply that $V(-\Delta+1)^{-1} Q_{1}$ is, since $(-\Delta+1)^{-1} Q_{1}$ and $\sum_{n \in I} \int_{\mathbb{B}} d k\left(E_{n}(k)-E\right) P_{n}(k)$ are bounded

$$
V(-\Delta+1)^{-1} Q_{1}=V(y) \frac{1}{1+|\boldsymbol{y}|^{3 / 2+\varepsilon}}\left(1+|\boldsymbol{y}|^{3 / 2+\varepsilon}\right)(-\Delta+1)^{-1} Q_{1} .
$$

Now consider $\left(1+|y|^{3 / 2+\varepsilon}\right)(-\Delta+1)^{-1} Q_{1}$ and prove it is bounded from $L^{2}$ to $L^{\infty}$; in fact:

$$
\begin{aligned}
& \sup _{\boldsymbol{y}}\left|\left(1+|\boldsymbol{y}|^{3 / 2+\varepsilon}\right)\right|\left|\int \frac{e^{-|\boldsymbol{x}-\boldsymbol{y}|}}{|\boldsymbol{x}-\boldsymbol{y}|} \frac{1}{1+|\boldsymbol{x}|^{3 / 2+\varepsilon}} \varphi(\boldsymbol{x}) d^{3} x\right| \\
& \leqq \sup _{\boldsymbol{y}} \int\left(1+|\boldsymbol{y}|^{3 / 2+\varepsilon}\right) \frac{e^{-|\boldsymbol{x}-\boldsymbol{y}|}}{|\boldsymbol{x}-\boldsymbol{y}|} \frac{1}{1+|\boldsymbol{x}|^{3 / 2+\varepsilon}}|\varphi(\boldsymbol{x})| d^{3} x .
\end{aligned}
$$

As

$$
\begin{aligned}
& 1+|\boldsymbol{y}|^{3 / 2+\varepsilon} \leqq 1+(|\boldsymbol{y}-\boldsymbol{x}|+|\boldsymbol{x}|)^{3 / 2+\varepsilon} \leqq 1+[2 \max (|\boldsymbol{y}-\boldsymbol{x}|,|\boldsymbol{x}|)]^{3 / 2+\varepsilon} \\
& \leqq 1+2^{3 / 2+\varepsilon} \max \left(|\boldsymbol{y}-\boldsymbol{x}|^{3 / 2+\varepsilon},|\boldsymbol{x}|^{3 / 2+\varepsilon}\right) \leqq 1+2^{3 / 2+\varepsilon}\left(|\boldsymbol{y}-\boldsymbol{x}|^{3 / 2+\varepsilon}+|\boldsymbol{x}|^{3 / 2+\varepsilon}\right) .
\end{aligned}
$$

Then, denoting $2^{3 / 2+\varepsilon}=\alpha$

$$
\begin{aligned}
& \sup _{\boldsymbol{y}} \ldots \leqq \int\left[1+\alpha\left(|\boldsymbol{y}-\boldsymbol{x}|^{3 / 2+\varepsilon}+|\boldsymbol{x}|^{3 / 2+\varepsilon}\right)\right] \frac{e^{-|\boldsymbol{x}-\boldsymbol{y}|}}{|\boldsymbol{x}-\boldsymbol{y}|} \frac{1}{1+|\boldsymbol{x}|^{3 / 2+\varepsilon}}|\varphi(\boldsymbol{x})| d^{3} x=A+B+C \\
& A=\int \frac{e^{-|\boldsymbol{x}-\boldsymbol{y}|}}{|\boldsymbol{x}-\boldsymbol{y}|} \frac{1}{1+|\boldsymbol{x}|^{3 / 2+\varepsilon}}|\varphi(\boldsymbol{x})| d^{3} x \leqq\left\|\frac{e^{-|\boldsymbol{x}|}}{|\boldsymbol{x}|}\right\|_{2}\|\varphi\|_{2} \\
& B=\alpha \int e^{-|\boldsymbol{x}-\boldsymbol{y}|}|\boldsymbol{x}-\boldsymbol{y}|^{1 / 2+\varepsilon} \frac{1}{1+|\boldsymbol{x}|^{3 / 2+\varepsilon}}|\varphi(\boldsymbol{x})| d^{3} z \leqq \alpha\left\||\boldsymbol{x}|^{1 / 2+\varepsilon} e^{-|\boldsymbol{x}|}\right\|_{2}\|\varphi\|_{2} \\
& C=\alpha \int \frac{e^{-|\boldsymbol{x}-\boldsymbol{y}|}}{|\boldsymbol{x}-\boldsymbol{y}|} \frac{|\boldsymbol{x}|^{3 / 2+\varepsilon}}{1+|\boldsymbol{x}|^{3 / 2+\varepsilon}}|\varphi(\boldsymbol{x})| d^{3} z \leqq \alpha\left\|\frac{e^{-|\boldsymbol{x}|}}{|\boldsymbol{x}|}\right\|_{2}\|\varphi\|_{2} .
\end{aligned}
$$

Then $\left\|\left(1+|y|^{3 / 2+\varepsilon}\right)(-\Delta+1)^{-1} Q_{1}\right\|_{\infty} \leqq M\|\varphi\|_{2}$.

Now return to the operator: $V(y) \frac{1}{1+|\boldsymbol{y}|^{3 / 2+\varepsilon}}\left(1+|\boldsymbol{y}|^{3 / 2+\varepsilon}\right)(-\Delta+1)^{-1} Q_{1}$

$$
\begin{aligned}
& \left\|V(\boldsymbol{y}) \frac{1}{1+|\boldsymbol{y}|^{3 / 2+\varepsilon}}(1+|\boldsymbol{y}|)^{3 / 2+\varepsilon} \int \frac{e^{-|\boldsymbol{x}-\boldsymbol{y}|}}{|\boldsymbol{x}-\boldsymbol{y}|} \frac{1}{1+|\boldsymbol{x}|^{3 / 2+\varepsilon}} \varphi(x) d^{3} x\right\|_{2}^{2} \\
& =\int d^{3} y|V(\boldsymbol{y})|^{2} \frac{1}{\left(1+|\boldsymbol{y}|^{3 / 2+\varepsilon}\right)^{2}}|\theta(\boldsymbol{y})|^{2} \\
& \leqq M^{2}\|\varphi\|_{2}^{2} \int_{\square} d^{3} y_{0}\left|V\left(\boldsymbol{y}_{0}\right)\right|^{2} \sum_{\boldsymbol{R}} \frac{1}{\left(1+\left|\boldsymbol{y}_{0}+\boldsymbol{R}\right|^{3 / 2+\varepsilon}\right)^{2}} \cdot\left(1^{\prime}\right) .
\end{aligned}
$$

If $m=1+\sum_{\boldsymbol{R}} \frac{1}{\left(1+|| \boldsymbol{R}|-d|^{3 / 2+\varepsilon}\right)^{2}} \Rightarrow\left(1^{\prime}\right) \leqq m M^{2}\left(\int_{\square} d^{3} y_{0}\left|V\left(\boldsymbol{y}_{0}\right)\right|^{2}\right)\|\varphi\|_{2}^{2}$. (d is the diameter of the unit cell, $\square$.) 
Then $V(-\Delta+1)^{-1} Q_{1}$ is bounded from, $L^{2}$ to $L^{2}$.

The continuity of $Q_{2}\left(1-P_{I}\right)\left(H_{B}-E\right)^{-1} Q_{1}$ results of the analyticity of $\left(1-P_{I}\right)\left(H_{B}-E\right)^{-1}$ on the interval $\Lambda$.

Now return to the proof of Theorem 1.2.

1) First of all we have to prove that we can define an operator, $H=H_{B}+Q$, self-adjoint. $Q=Q_{1} Q_{2}$ is relatively compact with respect to $H_{B}$ in fact:

$$
Q\left(H_{B}+1\right)^{-1}=Q(-\Delta+1)^{-1}-Q(-\Delta+1)^{-1} V\left(H_{B}+1\right)^{-1} .
$$

As $Q_{2}(-\Delta+1)^{-1}$ is compact and $Q_{1}$ is bounded, $Q(-\Delta+1)^{-1}$ is also compact; $V\left(H_{B}+1\right)^{-1}$ is bounded since it is closed and defined everywhere because $\mathscr{D}(V) \supset$ $\mathscr{D}(-\Delta)=\mathscr{D}\left(H_{B}\right)$ (cf. Avron, Grossmann, and Rodriguez [14]) and $\mathscr{R}\left(H_{B}+1\right)^{-1}=$ $\mathscr{D}\left(H_{B}\right)$. Then $Q\left(H_{B}+1\right)^{-1}$ is compact. So we can define a self-adjoint operator $H=H_{B}+Q$ the domain of which is $\mathscr{D}(-\Delta)$.

2) How to choose $\Gamma$ ?

$A(\lambda) \equiv Q_{2}\left(H_{B}-\lambda\right)^{-1} Q_{1}$ is a compact operator valued function analytic in the upper half-plane and it has an extension to a function continuous on the interval $A$ except at the points belonging to $\mathscr{E}_{c}^{-}$. As $\left(1+A\left(E_{ \pm}\right)\right)^{-1}$ exists when $E$ is real and sufficiently negative $(\|A(E)\| \rightarrow 0$ as $E \rightarrow-\infty)$ by Fredholm theorem the set $\mathscr{S}_{\boldsymbol{I}}$ where $\left(1+A\left(E_{ \pm}\right)\right)^{-1}$ does not exist is closed and has measure zero in $\Lambda-\mathscr{E}_{\boldsymbol{c}, \boldsymbol{I}}^{-}$ (cf. Simon [9], p. 127).

Then if we choose $E \in \Lambda-\left(\mathscr{S}_{\mathbf{I}} \cup \mathscr{E}_{c, I}^{-}\right)$, Conditions 1.2 and 1.3 are satisfied. All has been done for $Q_{2}\left(H_{B}-\lambda\right)^{-1} Q_{1}$ in Lemmas 1.3 and 1.4 can be repeat in an easier manner for $Q_{1}\left(H_{B}-\lambda\right)^{-1} Q_{1}$ hence Condition 1.1 is also satisfied if $E \in \Lambda-$ $\left(\mathscr{S}_{I} \cup \mathscr{E}_{c, \boldsymbol{I}}^{-}\right)$.

As $\Lambda$ is in some sense arbitrary, $\Gamma$ can be chosen in $\mathbb{R}^{1}-\left(\mathscr{S} \cup \mathscr{E}_{c}^{-}\right)$where $\mathscr{S}=\bigcup_{I} \mathscr{S}_{I}$ and $\mathscr{E}_{c}^{-}$is a subset of all the critical energies. Q.E.D.

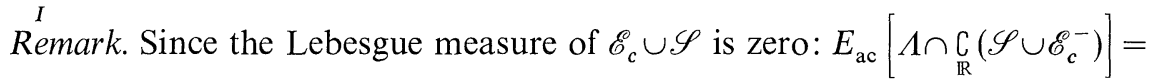
$E_{\mathrm{ac}}(\Lambda)$. Furthermore, $E_{B, \mathrm{ac}}(\Lambda)=E_{B}(\Lambda)$ since the spectrum of $H_{B}$ is absolutely continuous (cf. Thomas [10]), then:

$E_{\text {ac }}(\Lambda) H$ is unitarily equivalent to $E_{B}(\Lambda) H_{B}, \forall \Lambda \subset \mathbb{R}$.

\section{Eigenfunction Expansion}

Suppose in addition to the Conditions I.1-3 the following ones hold for a Borel set $\Gamma$ of $\mathbb{R}$.

Condition II.1. There exists a $\sigma$-finite measure space $(\Omega, \Sigma, \varrho)$, a partial isometry $U_{0}$ of $\mathscr{H}$ onto $L^{2}(\varrho)$ with initial set $\mathscr{H}_{0, \text { ac }}$ and a measurable function $\omega: \Omega \rightarrow \Gamma$ such that:

$$
\left(U_{0} E_{0, \text { ac }}(\Delta) f\right)(\xi)=\chi_{\Delta}(\omega(\xi))\left(U_{0} f\right)(\xi) \varrho \cdot \text { a.e.; } \xi \in \Omega
$$

for each $f \in \mathscr{H}$ and for every Borel set $\Delta \subset \mathbb{R}$. $\chi_{\Delta}$ is the characteristic function of $\Delta$.

Condition II.2. There exists a mapping $\Psi_{0}: \Omega \rightarrow \mathscr{H}^{-}=\left(\mathscr{H}^{+}\right)^{*}$ such that

$$
\left(U_{0} f\right)(\xi)=\left\langle\Psi_{0}(\xi), f\right\rangle \varrho \cdot \text { a.e. } \xi \in \Omega \text { and for all } f \in \mathscr{H}^{+} .
$$


In our case $\Omega=\mathbb{Z}^{+} \times \mathbb{B}$; $\varrho$ is the product measure of the pure point measure of $\mathbb{Z}^{+}$ and the natural measure on $\mathbb{B}$. $U_{0}$ is the unitary operator $U_{B}$ (see Footnote ${ }^{3}$ ) and $\omega$ is precisely the energy band function $(n, k) \in \Omega \rightarrow E_{n}(k)$. The mapping $\psi_{0}$ can be defined from the equality

$$
\left(U_{B} f\right)(n, k)=\int \psi_{n k}^{B^{*}}(\boldsymbol{x}) f(\boldsymbol{x}) d^{3} x
$$

then $\quad \psi_{0}=\psi^{B}:(n, k) \rightarrow \psi_{n \boldsymbol{k}}^{B} \quad$ (Bloch function) $\psi_{n \boldsymbol{k}}^{B}(\boldsymbol{x})=\sum_{\boldsymbol{K}} c_{n}^{\boldsymbol{k}}(\boldsymbol{k}) \exp (i(\boldsymbol{k}+\boldsymbol{K}) \boldsymbol{x})$, as $\sum_{\mathbf{K}}\left|c_{n}^{\boldsymbol{K}}(k)\right|<+\infty$ then $\psi_{n \boldsymbol{k}}^{B} \in L^{\infty} \subset \mathscr{H}^{-}$.

Now Kato and Kuroda proved the following theorem (written here with our notations).

Theorem II.1. Suppose that Conditions (I.1-I.3) hold for some Borel set $\Gamma \subset \mathbb{R}$. Assume further that the operator $H_{B}$ satisfies Conditions (II.1 and II.2) for the same $\Gamma$.

Then the operator $H=H_{B}+Q$ satisfies these Conditions (II.1 and II.2) with the same space measure and the same measurable function $\omega$.

More precisely we have

(1) The operators $U_{ \pm}=U_{B} W_{ \pm}^{*}$ are partial isometries of $L^{2}\left(\mathbb{R}^{3}\right)$ onto $L^{2}\left(\mathbb{Z}^{+} \times \mathbb{B}\right)$ with initial set $\mathscr{H}_{\mathrm{ac}}(\Gamma)$. Furthermore we have:

$\left(U_{ \pm} E(\Delta) f\right)(n, k)=\chi_{\Delta}\left(E_{n}(k)\right)\left(U_{ \pm} f\right)(n, k) \quad$ for a.e. $\quad(n, k) \in \mathbb{Z}^{+} \times \mathbb{B}$.

(2) The following equation in $\mathscr{H}^{+}=\left\{f \mid\left(1+|\boldsymbol{x}|^{3 / 2+\varepsilon}\right) f \in L^{2}\left(\mathbb{R}^{3}\right)\right\}$

$$
\left[1+Q\left(H_{B}-E_{n}(\hat{k}) \pm i 0\right)^{-1}\right]^{*} \psi_{n k}^{ \pm}=\psi_{n k}^{B}
$$

has a unique solution $\psi_{n \boldsymbol{k}}^{ \pm} \in \mathscr{H}^{-}$for every $(n, k) / E_{n}(k) \in \Gamma$ such that

$$
\left(U_{ \pm} f\right)(n, k)=\left\langle\psi_{n k}^{ \pm}, f\right\rangle \text { a.e. }(n, k) \in \mathbb{Z}^{+} \times \mathbb{B}
$$

and for every $f \in \mathscr{H}^{+}$.

Remark. The Eq. (II.1) can be written

$$
\psi_{n \boldsymbol{k}}^{ \pm}(\boldsymbol{x})=\psi_{n \boldsymbol{k}}^{B}(\boldsymbol{x})-\int G_{B}\left(\boldsymbol{x}, \boldsymbol{y}, E_{n}(k) \pm i 0\right) Q(\boldsymbol{y}) \psi_{n \boldsymbol{k}}^{ \pm}(\boldsymbol{y}) d^{3} y
$$

and is called the Lippmann-Schwinger equation.

\section{Spectral Properties of $H$ when $Q$ has an Exponential Decay}

If we add to the precedent conditions on $Q$ the hypothesis $Q$ has an exponential decay, we can say more about the set of points in $\mathbb{R}-\mathscr{C}_{c}^{-}$such that $(1+A(E))^{-1}$ do not exist, i.e. about the eigenvalues imbedded in the continuum and the singular continuous spectrum.

Theorem. Let $Q$ and $V \in L^{2, \operatorname{loc}_{(}}\left(\mathbb{R}^{3}\right)$ and $\int_{|\boldsymbol{x}|>M}|Q(\boldsymbol{x})|^{2} e^{\alpha|x|} d^{3} x<+\infty$ for some $\alpha>0$ and $M>0$, then in a finite interval $\left[E_{1}, E_{2}\right]$ without critical energies in which 
there is no band intersection ${ }^{5}$, the singular continuous spectrum is empty and the number of eigenvalues is finite.

Proof. Let $Q_{1}^{\prime}(\boldsymbol{y})=e^{-\alpha / 4|\boldsymbol{y}|} /\left(1+|\boldsymbol{y}|^{3 / 2+\varepsilon}\right)$ and $Q_{1}^{\prime} Q_{2}^{\prime}=Q$.

We want to define an extension of operator $Q_{2}^{\prime} P^{\prime}\left(H_{B}-E\right)^{-1} Q_{1}^{\prime}$ for $E$ going through the real axis to the non physical sheet, $P^{\prime}=\sum_{n \in I^{\prime}} \int_{n}^{\oplus} P_{n}(k) d^{3} k . I^{\prime}$ is the set of all the $n$ such that $E_{n}(k) \in\left[E_{1}, E_{2}\right]$ for some $k \in \mathbb{B}$. To simplify the text we shall, in the following, only consider a band. As we discuss in Appendix A, introducing new variables $(E, \theta, \varphi)$ in place of $\left(k_{1}, k_{2}, k_{3}\right)$ we can rewrite

$$
G_{B}^{0}(\boldsymbol{x}, \boldsymbol{y}, \lambda)=\int_{E_{1}}^{E_{2}} d E \frac{1}{E-\lambda} \int_{0}^{2 \pi} d \varphi \int_{0}^{\pi} d \theta\left(\psi_{n \boldsymbol{x}} \psi_{n \boldsymbol{y}}^{*} J_{n}\right)(E, \theta, \varphi)
$$

We define now an extension of $G_{B}^{0}(\boldsymbol{x}, \boldsymbol{y}, \lambda)$ by distording the contour of integration over $E$; more precisely the new contours are $\mathscr{C}_{\alpha}=\left[\lambda^{\prime} \in \mathbb{C} \mid \lambda^{\prime}=E-i \alpha \Theta(E)\right]$ where $\Theta$ is a continuous positive function such that $\Theta\left(E_{1}\right)=\Theta\left(E_{2}\right)=0, \alpha$ is a parameter varying from 0 to 1 .

$\Theta$ is chosen so that $\mathscr{C}_{1}$ is the frontier of $\mathscr{D}$ (see Footnote $\left.{ }^{5}\right)$; as $\psi_{n \boldsymbol{x}}(\lambda ; \theta, \varphi)$ and $J_{n}(\lambda, \theta, \varphi)$ are analytic with respect to $\lambda$, if $\operatorname{Im} \lambda>0$ the extension $G_{B, \alpha}^{0}$ is equal to $G_{B}^{0} \equiv G_{B, 0}^{0}$, so $G_{B, \alpha}^{0}$ is the analytic continuation of $G_{B}^{0}$ to some $\lambda \in \mathscr{D}$ with $\operatorname{Im} \lambda<0$.

We prove now that the operator the kernel of which is:

$$
Q_{2}^{\prime}(\boldsymbol{x}) G_{B, 1}^{0}(\boldsymbol{x}, \boldsymbol{y}, \lambda) Q_{1}^{\prime}(\boldsymbol{y})
$$

is Hilbert-Schmidt.

$$
G_{B, 1}^{0}(\boldsymbol{x}, \boldsymbol{y}, \lambda)=G_{B, 0}^{0}(\boldsymbol{x}, \boldsymbol{y}, \lambda)+\int_{\mathscr{C}^{\prime}} d E \frac{1}{E-\lambda} \int_{0}^{2 \pi} d \varphi \int_{0}^{\pi} d \theta\left(\psi_{n x} \psi_{n y}^{*} J_{n}\right)(E, \theta, \varphi)
$$

as $\int_{\mathscr{C}_{1}} d E \ldots=\int_{\mathscr{C}_{0}} d E \ldots+\int_{\mathscr{C}^{\prime}} d E \ldots$

Fig. 2

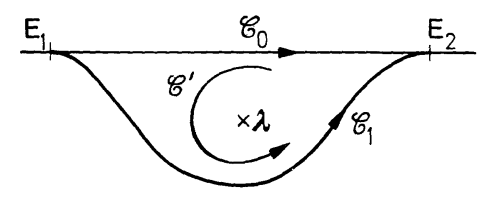

Then utilizing the Cauchy theorem:

$$
G_{B, 1}^{0}(\boldsymbol{x}, \boldsymbol{y}, \lambda)=G_{B, 0}^{0}(\boldsymbol{x}, \boldsymbol{y}, \lambda)+\int_{0}^{2 \pi} d \varphi \int_{0}^{\pi} d \theta\left(\psi_{n \boldsymbol{x}} \psi_{n \boldsymbol{y}}^{*} J_{n}\right)(\lambda, \theta, \varphi) .
$$

Now using the continuity of $\psi_{n x} \psi_{n y}^{*} J_{n}$ with respect to $\theta$ and $\varphi$

$$
G_{B}^{0}(\boldsymbol{x}, \boldsymbol{y}, \lambda)=G_{B, 0}^{0}(\boldsymbol{x}, \boldsymbol{y}, \lambda)+2 \pi^{2}\left(\psi_{n \boldsymbol{x}} \psi_{n \boldsymbol{y}}^{*} J_{n}\right)\left(\lambda, \theta_{0}, \varphi_{0}\right) .
$$

\footnotetext{
$5 \quad \exists k$ such that $E_{n}(k)=E_{n^{\prime}}(k) \in\left[E_{1}, E_{2}\right]$ and $n \neq n^{\prime}$. As a consequence the eigenvalues of $H_{B}^{k}$ belonging to $\left[E_{1}, E_{2}\right]$ and the corresponding eigenvectors are real holomorphic in $\mathbb{B}$ and this holomorphy can be extended on some domain $\mathscr{D}$ of the complex space $\mathbb{C}^{3}$.
} 
Let $k\left(\lambda, \theta_{0}, \varphi_{0}\right)$ be denoted $k_{0} ; \psi_{n \boldsymbol{x}}\left(\lambda, \theta_{0}, \varphi_{0}\right)=\sum_{\boldsymbol{K}} c_{n}^{\mathbf{K}}\left(\boldsymbol{k}_{0}\right) e^{i\left(k_{0}+K\right) x}$

Then

$$
\left|\psi_{n \boldsymbol{x}}\left(\lambda, \theta_{0}, \varphi_{0}\right)\right| \leqq e^{-\operatorname{Im}\left(\boldsymbol{k}_{0}, \boldsymbol{x}\right)} \sum_{\boldsymbol{K}}\left|c_{n}^{\boldsymbol{K}}\left(\boldsymbol{k}_{0}\right)\right|
$$

So

$$
\begin{aligned}
& \iint d^{3} \boldsymbol{x} d^{3} \boldsymbol{y}\left|Q_{2}^{\prime}(\boldsymbol{x})\right|^{2}\left|Q_{1}^{\prime}(\boldsymbol{y})\right|^{2}\left|G_{B, 1}^{0}(\boldsymbol{x}, \boldsymbol{y}, \lambda)\right|^{2} \\
& \leqq \\
& \quad 2 \int d^{3} x d^{3} y\left|Q_{2}^{\prime}(\boldsymbol{x})\right|^{2}\left|Q_{1}^{\prime}(\boldsymbol{y})\right|^{2}\left|G_{B, 0}^{0}(\boldsymbol{x}, \boldsymbol{y}, \lambda)\right|^{2} \\
& \quad+4 \pi^{2}\left|J_{n}\left(k_{0}\right)\right|^{2}\left(\sum_{\boldsymbol{K}}\left|c_{n}^{\mathbf{K}}\left(k_{0}\right)\right|\right)^{2} \iint\left|Q_{2}^{\prime}(\boldsymbol{x})\right|^{2}\left|Q_{1}^{\prime}(\boldsymbol{y})\right|^{2} e^{-\operatorname{Im} \boldsymbol{k}_{0}(\boldsymbol{x}-\boldsymbol{y})} d^{3} x d^{3} y .
\end{aligned}
$$

The first term is finite (the proof exactly follows as in Lemma 1.3). The second term is also finite since $\sum_{\boldsymbol{K}}\left|c_{n}^{\boldsymbol{K}}\left(k_{0}\right)\right|<+\infty$ (see Appendix B), and:

$$
\begin{aligned}
& \int\left|Q_{1}^{\prime}(\boldsymbol{y})\right|^{2} e^{\operatorname{Im} \boldsymbol{k}_{0} \cdot \boldsymbol{y}} d^{3} y \leqq \int\left|Q_{1}^{\prime}(\boldsymbol{y})\right|^{2} e^{\left|\operatorname{Im} \boldsymbol{k}_{0}\right| \cdot|\boldsymbol{y}|} d^{3} y<+\infty \\
& \int\left|Q_{2}^{\prime}(\boldsymbol{x})\right|^{2} e^{-\operatorname{Im} \boldsymbol{k}_{0}, \boldsymbol{x}} d^{3} x \leqq \int\left|Q_{2}^{\prime}(\boldsymbol{x})\right|^{2} e^{\left|\operatorname{Im} \boldsymbol{k}_{0}\right| \cdot|\boldsymbol{x}|} d^{3} x \\
& =\int_{|\boldsymbol{x}|<M}|Q(\boldsymbol{x})|^{2}\left(1+|\boldsymbol{x}|^{3+2 \varepsilon}\right) e^{\alpha / 2|\boldsymbol{x}|} e^{\left|\operatorname{Im} \boldsymbol{k}_{0}\right| \cdot|\boldsymbol{x}|} d^{3} x \\
& \quad+\int_{|\boldsymbol{x}|>M}|Q(\boldsymbol{x})|^{2}\left(1+|\boldsymbol{x}|^{3+2 \varepsilon}\right) e^{\alpha / 2|\boldsymbol{x}|} e^{\left|\operatorname{Im} \boldsymbol{k}_{0}\right| \cdot|\boldsymbol{x}|} d^{3} x .
\end{aligned}
$$

The first term is finite since $Q \in L^{2, l o c}\left(\mathbb{R}^{3}\right)$, the second also by hypothesis if $\left|\operatorname{Im} \boldsymbol{k}_{0}\right|<\alpha / 2$. So in the two cases it is sufficient to choose $\lambda$ sufficiently near the real axis so that $\left|\operatorname{Im} \boldsymbol{k}_{0}\right|<\alpha / 2$. So the extended operator $A_{1}(E)$ is compact and the conclusion follows immediately.

Acknowledgements. I wish to thank Prof. J. C. Guillot to call my attention to the work of KatoKuroda and Prof. A. Grossmann and E. Mourre for many valuable discussions.

\section{Appendix A}

In Avron, Grossmann, and Rodriguez [14], it is shown that the spectrum of the reduced Bloch hamiltonian $H_{B}^{k}$ consists of real isolated points of finite multiplicity $E_{n}(k)$; the $E_{n}$ can be chosen holomorphic in any one of the variables $k_{i}$ (but they will not in general be single valued as a function of all three variables $k_{i}$ ).

Thomas proved, as a consequence, that the measure of the set of points $k \in B$ such that $E_{n}(k)$ equals a fixed energy $E$ is zero. In the same manner it can be proven that the measure of the set $\mathscr{E}_{c}$ (critical points) is zero. We can also deduce that critical points can be isolated or distributed along a curve or a surface of equal energy.

If we diagonalize the matrix of the second partial derivatives at the critical point we distinguish two principal sorts of critical points, extremal point if all the signs of the $\partial^{2} E_{n} / \partial k_{i}^{2}$ are the same, saddle point if the signs are different. 
Now state the theorem

Theorem A.1. Let $E^{\prime}$ belong to the bands the index of which is in the set $\boldsymbol{I}$ and

$$
g_{\boldsymbol{R}}\left(\boldsymbol{x}_{0}, \boldsymbol{y}_{0}, E^{\prime} \pm i 0\right)=\lim _{\varepsilon \rightarrow 0} \sum_{n \in \boldsymbol{I}} \iiint_{\mathbb{B}} d^{3} k \frac{\psi_{n k}\left(\boldsymbol{x}_{0}\right) \psi_{n k}^{*}\left(\boldsymbol{y}_{0}\right)}{E_{n}(k)-E^{\prime} \pm i \varepsilon}
$$

then $\left|g_{\boldsymbol{R}}\left(\boldsymbol{x}_{0}, \boldsymbol{y}_{0}, E^{\prime} \pm i 0\right)\right| \leqq \alpha\left(E^{\prime}\right) /|\boldsymbol{R}|$ when $|\boldsymbol{R}|$ is sufficiently large, when $E$ is not $a$ saddle critical point.

Proof. For each $n \in \boldsymbol{I}$ divide the Brillouin zone in domains $\mathscr{D}_{n}^{i}$, whose frontiers are the curves of constant energy: $E_{n}^{S_{i}}\left(E_{n}^{S_{i}}\right.$ denote the critical value taken by $E_{n}$ at the saddle point $k_{s_{1}}$.

Except possibly at the extremal points and at the "fluted points" [19] we define a diffeomorphism by associating to each $k \in \mathscr{D}_{n}^{i}$ the corresponding energy $E=E_{n}(k)$ and the angular coordinates $\theta, \varphi$ in fact the matrix derivative of such a transform $\mathscr{E}_{n}^{i}$, has a determinant equal to $\nabla E_{n}\left(k-k_{n}^{i}\right) /\left|k-k_{n}^{i}\right|^{3} \sin \theta$ where $k_{n}^{i}$ is the "center" of the new coordinates (the $\boldsymbol{R}$ direction is taken as the $z$-axis).

Let $J_{n}^{i}(E, \theta, \varphi)=\left|k-k_{n}^{i}\right|^{3} \sin \theta / \nabla E_{n} \cdot\left(k-k_{n}^{i}\right)$ denote the Jacobian of $\left(\mathscr{E}_{n}^{i}\right)^{-1}$ at the point $E, \theta, \varphi ; E_{n}^{i}, \dot{E}_{n}^{i}$ resp. the $\inf _{\boldsymbol{k} \in \mathscr{D}_{n}^{i}}(\sup ) E_{n}(k)$ and $\kappa=k-k_{n}^{i}$.

Then:

$$
\begin{aligned}
g_{\boldsymbol{R}}\left(\boldsymbol{x}_{0}, \boldsymbol{y}_{0}, E^{\prime} \pm i 0\right)= & \lim _{\varepsilon \rightarrow 0} \sum_{n \in I} \sum_{i} \int_{E_{n}^{i}}^{\dot{E}_{n}^{i}} d E \frac{1}{E-E^{\prime} \mp i \varepsilon} \\
& \cdot \int_{0}^{2 \pi} d \varphi \int_{0}^{\pi} d \theta\left(\psi_{n x} \psi_{n y}^{*} J_{n}^{i}\right)(E, \theta, \varphi) e^{i|\kappa||\boldsymbol{R}| \cos \theta} .
\end{aligned}
$$

We can separate the summation in two parts considering in one side the $n$ and $i$ such that $\left.E^{\prime} \in\right] E_{n}^{i}, \dot{E}_{n}^{i}\left[\right.$ and on the other side $m$ and $j$ such that $\left.E^{\prime} \notin\right] E_{m}^{j}, \dot{E}_{m}^{j}[$. In the following we shall write only one term of each type to simplify the text.

Denote

$$
f_{n}^{i}(E, \boldsymbol{R})=\int_{0}^{2 \pi} d \varphi \int_{0}^{\pi} d \theta\left(\psi_{n x} \psi_{n y}^{*} J_{n}^{i}\right)(E, \theta, \varphi) e^{i|\kappa||\boldsymbol{R}| \cos \theta},
$$

it is differentiable with respect to $E$ in the interval $] E_{n}^{i}, \dot{E}_{n}^{i}[$; then by the mean value theorem the term (1) in:

$$
\begin{aligned}
g_{\boldsymbol{R}}\left(\boldsymbol{x}_{0}, \boldsymbol{y}_{0}, E^{\prime} \pm i 0\right)= & \lim _{\varepsilon \rightarrow 0} \int_{E_{n}^{i}}^{\dot{E}_{n}^{h}} \frac{f_{n}^{i}(E, \boldsymbol{R})-f_{n}^{i}\left(E^{\prime}, \boldsymbol{R}\right)}{E-E^{\prime} \neq i \varepsilon} d E \\
& +\lim _{\varepsilon \rightarrow 0} f_{n}^{i}\left(E^{\prime}, \boldsymbol{R}\right) \int_{E_{n}^{i}}^{\dot{E}_{n}^{i}} \frac{d E}{E-E^{\prime} \mp i \varepsilon}+\int_{E_{m}^{\prime}}^{\dot{E}_{m}^{j}} d E f_{m}^{j}(E, \boldsymbol{R}) /\left(E-E^{\prime}\right)
\end{aligned}
$$

becomes:

$$
\int_{E_{n}^{i}}^{\dot{E}_{n}^{i}} \frac{\partial f_{n}^{i}\left(E^{\prime}+\theta_{E^{\prime}}(E), \boldsymbol{R}\right)}{\partial E} d E=f_{n}^{i}\left(E^{\prime}+\theta_{E^{\prime}}\left(\dot{E}_{n}^{i}\right), \boldsymbol{R}\right)-f_{n}^{i}\left(E^{\prime}+\theta_{E^{\prime}}\left(E_{n}^{i}\right), \boldsymbol{R}\right) ;
$$

$\theta_{E^{\prime}}$ is a continuous function the values of which are in the interval $] E_{n}^{i}-E^{\prime}, \dot{E}_{n}^{i}-E^{\prime}[$. 
The term (2) equals $f_{n}^{i}\left(E^{\prime}, \boldsymbol{R}\right) \log \frac{\dot{E}_{n}^{i}-E^{\prime}}{E_{n}^{i}-E^{\prime}}$ and term (3)

$f_{m}^{j}\left(E_{m}^{j}+\alpha\left(E^{\prime}\right)\right) \int_{E_{m}^{j}}^{\dot{E}_{m}^{j}} d E 1 /\left(E-E^{\prime}\right)$ with $\alpha\left(E^{\prime}\right)>0$.

So we have to study $f_{n}^{i}(E, \boldsymbol{R})$ as a function of $\boldsymbol{R}$ whenever $\left.E \in\right] E_{n}^{i}, \dot{E}_{n}^{i}[$ is.

If $|\boldsymbol{R}| \neq 0$ we can rewrite $f_{n}^{i}(E, \boldsymbol{R})$ in the form:

$$
f_{n}^{i}(E, \boldsymbol{R})=\int_{0}^{2 \pi} d \varphi \int_{0}^{\pi} d \theta \frac{\partial}{\partial \theta} e^{i|\kappa||\boldsymbol{R}| \cos \theta}\left(\psi_{n x} \psi_{n y}^{*} J_{n}^{i}\right)(E, \theta, \varphi) \frac{1}{i|\boldsymbol{R}| \frac{\partial}{\partial \theta}|\kappa| \cos \theta}
$$

and integrate by parts

$$
\begin{aligned}
f_{n}^{i}(E, R)= & 1 /(i|\boldsymbol{R}|) \int_{0}^{2 \pi} d \varphi\left|\frac{e^{i|\kappa||\boldsymbol{R}| \cos \theta} \psi \psi^{*} J_{n}^{i}}{\frac{\partial}{\partial \theta}|\kappa| \cos \theta}\right|_{\theta=0}^{\theta=\pi}-1 /(i|\boldsymbol{R}|) \int_{0}^{2 \pi} d \varphi \int_{0}^{\pi} d \theta e^{i|\kappa||\boldsymbol{R}| \cos \theta} \\
& \cdot \frac{\partial}{\partial \theta} \frac{\left(\psi \psi^{*} J_{n}^{i}\right)(E, \theta, \varphi)}{\frac{\partial}{\partial \theta}|\kappa| \cos \theta} .
\end{aligned}
$$

It is now sufficient to prove that the two integrals exist and are majorized by a function of $E$ only. As

$$
J_{n}^{i}(E, \theta, \varphi) / \frac{\partial}{\partial \theta}|\kappa| \cos \theta=-|\kappa| \sin \theta /\left(\frac{\partial E}{\partial|\kappa|} \sin \theta+\frac{\partial E}{\partial \theta} \frac{1}{|\kappa|} \cos \theta\right) .
$$

If $\theta \rightarrow 0$ or $\pi$ we have to consider two cases:
a) $\frac{\partial E}{\partial \theta}(E, 0) \neq\left. 0 \Rightarrow \frac{J_{n}^{i}(E, \theta, \varphi)}{\frac{\partial}{\partial \theta}|\kappa| \cos \theta}\right|_{\theta=0, \pi}=0$
b) $\frac{\partial E}{\partial \theta}(E, 0)=\left.0 \Rightarrow \frac{J_{n}^{i}(E, \theta, \varphi)}{\frac{\partial}{\partial \theta}|\kappa| \cos \theta}\right|_{\theta=0, \pi}=-|\kappa|\left(\frac{\partial E}{\partial|\kappa|}(E, 0)\right)^{-1}$.

In the first case the first integral in $f_{n}^{i}(E, \boldsymbol{R})$ disappears, in the second it is equal to

$$
\frac{2 \pi}{i|\boldsymbol{R}|}\left[e^{i|\kappa||\boldsymbol{R}|} \psi \psi^{*}(E, 0) \frac{|\kappa|(E, 0)}{\frac{\partial E}{\partial|\kappa|}(E, 0)}-e^{-i|\kappa||\boldsymbol{R}|} \psi \psi^{*}(E, \pi) \frac{|\kappa|(E, \pi)}{\frac{\partial E}{\partial|\kappa|}(E, \pi)}\right] .
$$

Functions $\psi_{n}$ are bounded. $|\kappa|(E, 0) / \frac{\partial E}{\partial|\kappa|}(E, 0) \rightarrow+\infty$ only when $E$ goes to the $E_{n}^{i}$ or $\dot{E}_{n}^{i}$ which is the critical saddle point. Furthermore as $|\kappa|(E, 0) / \frac{\partial E}{\partial|\kappa|}(E, 0)$ is continuous it is bounded on a closed interval contained in $] E_{n}^{i}, \dot{E}_{n}^{i}[$. 
By some tricky calculation we obtain the same result from the second integral, then the conclusion follows immediately.

Theorem A.2. $g_{\boldsymbol{R}}\left(\boldsymbol{x}_{0}, \boldsymbol{y}_{0}, E^{\prime} \pm i 0\right)$ are continuous functions in $E^{\prime}$.

Proof. If $f(E, \boldsymbol{R})$ is an Holder continuous function with respect to $E$

$$
\lim _{\varepsilon \rightarrow 0} \int_{E_{n}^{i}}^{\dot{E}_{n}^{2}} f(E, \boldsymbol{R}) /\left(E-E^{\prime} \mp i \varepsilon\right) d E
$$

is also Holder continuous with the same order. Since $f(E, \boldsymbol{R})$ is differentiable in the interval $] E_{n}^{i}, \dot{E}_{n}^{i}[$, the result follows immediately.

\section{Appendix B. Properties of the Bloch Functions}

The improper eigenfunction of $H_{B}: \psi_{n k}(x)$ can be written in the form

$$
\psi_{n \boldsymbol{k}}(\boldsymbol{x})=\sum_{\boldsymbol{K}} c_{n}^{\boldsymbol{K}}(\boldsymbol{k}) e^{i(\boldsymbol{k}+\boldsymbol{K}) \boldsymbol{x}},
$$

where the sequence $\left\{c_{n}^{\mathbf{K}}(k)\right\} \in l^{2}$ is the $n^{\text {th }}$ eigenfunction of the reduced Bloch hamiltonian $H_{B}^{k}$.

$$
(k+\boldsymbol{K})^{2} c_{n}^{\mathbf{K}_{n}}(k)+\sum_{\mathbf{K}^{\prime}} \hat{V}_{\boldsymbol{K}-\boldsymbol{K}^{\prime}} c_{n}^{\boldsymbol{K}^{\prime}}(k)=E_{n}(k) c_{n}^{\boldsymbol{K}^{\mathbf{K}}}(k) .
$$

Lemma 3.1. For $k$ real

a) $\sum_{\mathbf{K}}\left|c_{n}^{\mathbf{K}}(k)\right|<+\infty$.

b) $\psi_{n \boldsymbol{k}}(\boldsymbol{x})$ is a bounded function in $\boldsymbol{x}$.

Proof. $c_{n}^{\mathbf{K}}(k)$ is an eigenfunction of $H_{B}^{k}$ then it belongs to $\mathscr{D}\left(H_{B}^{\boldsymbol{k}}\right)=\mathscr{D}\left(-\Delta^{\boldsymbol{k}}\right)$; then:

$$
\begin{aligned}
& \sum_{\boldsymbol{K}}\left|1+(k+\boldsymbol{K})^{2}\right|^{2}\left|c_{n}^{\boldsymbol{K}}(k)\right|^{2}<+\infty \\
& \sum_{\mathbf{K}}\left|c_{n}^{\boldsymbol{K}}(k)\right|=\sum_{\boldsymbol{K}}\left(1+(k+\boldsymbol{K})^{2}\right)^{-1}\left[1+(k+\boldsymbol{K})^{2}\right]\left|c_{n}^{\mathbf{K}}(k)\right| \\
& \leqq \sum_{\boldsymbol{K}^{\prime}}\left(1+\left(k+\boldsymbol{K}^{\prime}\right)^{2}\right)^{-2} \sum_{\boldsymbol{K}}\left[1+(k+\boldsymbol{K})^{2}\right]^{2}\left|c_{n}^{\boldsymbol{K}}(k)\right|^{2}<+\infty \quad \text { by } \quad \text { (B.1) }
\end{aligned}
$$

As $\left|\psi_{n \boldsymbol{k}}(x)\right| \leqq \sum_{\boldsymbol{K}}\left|c_{n}^{\boldsymbol{K}}(\boldsymbol{k})\right|$, b) follows immediately.

\section{References}

1. Kato,T., Kuroda,S.-T.: Theory of simple scattering and eigenfunction expansions. In: Functional analysis and related fields, Browder (Ed.). Berlin-Heidelberg-New York: Springer 1970

2. Kuroda,S.-T.: A stationary method of scattering and some applications. In: Proceedings of the Int. Conf. on Functional Analysis. University of Tokyo Press 1970

3. Kosicki-Paul, W.: Phys. Rev. Letters 17, 246-249 (1966); 17, 1175-1177 (1966)

4. Onton, A.: Phys. Rev. 186, 786-790 (1969); B 4, 4449-4452 (1971)

5. Bassani, F.: Theory of imperfect crystalline solids, Trieste lectures, p. 265. Vienna: IAEA 1970

6. Preziosi, B.: Nuovo Cimento 6 B, 131-138 (1971)

7. Bassani, F., Iadonisi, G., Preziosi, B. : Rept. Progr. Phys. 37, Part 3 (1974)

8. Ikebe, T.: Arch. Rat. Mech. Anal. 5, 1-34 (1960) 
9. Simon, B.: Quantum mechanics for Hamiltonians defined as quadratic forms. Princeton: Princeton University Press 1971

10. Thomas, L.: Commun. math. Phys. 33, 339 (1973)

11. Lenahan, T.A.: On quantum mechanical scattering in periodic media. Ph.D. Thesis, in Applied Mathematics. University of Pennsylvania (1970)

12. Troianiello, G.: J. Math. Phys. 15, 2048 (1974)

13. Guillot, J.-C.: Report of the Department of Mathematics. University of Paris-Nord (1974)

14. Avron, J., Grossmann, A., Rodriguez, R.: Rept. Math. Phys. 5, 113 (1974)

15. Kato, T.: Perturbation theory for linear operators. Berlin-Heidelberg-New York: Springer 1966

16. Balslev, E.: Math. Scand. 19, 193 (1966)

17. Avron, J., Grossmann, A., Rodriguez, R., Zak, J. : Preprint CNRS-C.P.T. 1975

18. Callaway, J.: J. Math. Phys. 5, 783-798 (1964)

19. Phillips, J.C.: Phys. Rev. 104, 1263 (1956)

Communicated by W. Hunziker

Received August 4, 1975 